\title{
Impact of FDA-Required Cardiovascular Outcome Trials on Type 2 Diabetes Clinical Study Initiation From 2008 to 2017
}

Therapeutic Innovation \& Regulatory Science 2020, Vol. 54(3) 640-644 (C) The Author(s) 2020 https://doi.org//0.1007/s4344I-019-00098-y

\author{
Cameron M. Kieffer, $\mathbf{P h D}^{\prime} \odot$, and Andrew S. Robertson, PhD, JD'®
}

\begin{abstract}
Background: Since 2008, the US Food and Drug Administration (FDA) has required that drug manufacturers conduct postmarket cardiovascular outcomes trials (CVOTs) for approved type 2 diabetes mellitus (T2DM) drugs. The utility and impact of these studies in determining atherosclerotic cardiovascular risk was reviewed during an FDA Advisory Committee Meeting held on October 24, 2018. Drug manufacturers and patient advocates at this meeting contended that the FDA-required CVOT studies discouraged private sector investment into developing novel T2DM drugs. Here, we explore these contentions by reviewing private sector investment in T2DM drug development from 2000 through 2008, followed by a deductive analysis of how associated events-including the implementation of the CVOT requirement-may have precipitated any observed changes.

Methods: We collected and analyzed industry-sponsored interventional trials for T2DM initiated between January I, 2000, and December 3I, 20I7, and compared observed trends with those seen across all trials, specific diseases, and against patent filings of novel antidiabetic agents.

Results: The analysis shows a marked decline in initiated T2DM clinical trials from 2008 through 2017. Possible influencing factors, including the 2008 financial crisis and a slow in the discovery of novel antidiabetic agents may have contributed, but could not fully account for this decline in T2DM studies.

Conclusions: These observations are consistent with the statements made by industry representatives and patient advocates at the 2018 Advisory Committee meeting. As the FDA reconsiders postmarket requirements for T2DM products, these observations underscore the importance of considering more efficient postmarket study structures to assess cardiovascular safety beyond mandatory CVOTs.
\end{abstract}

\section{Keywords}

cardiovascular outcomes trials, data-driven, postmarketing, regulatory science, drug development

\section{Introduction}

In December 2008, the FDA utilized their new authority under Section 505(o)(3) of the Food, Drug, and Cosmetics Act (FDCA) to require that manufacturers conduct additional studies to detect atherosclerotic cardiovascular (CV) risk for all prospective FDA-approved therapies for the treatment of type 2 diabetes mellitus (T2DM). ${ }^{1}$ This requirement was implemented by the FDA primarily in response to a then-reported $\mathrm{CV}$ safety concern regarding thiazolidinedione rosiglitazone (AVANDIA), a safety concern that has since been largely discredited. ${ }^{2-5}$

A significant component of this FDA requirement had sponsors conduct postmarket cardiovascular outcomes trails (CVOTs) on all newly approved products, regardless of risk. ${ }^{6}$ Since then, industry has invested significant resources and developed a decade of knowledge through investigating atherosclerotic CV risk for T2DM products, including the enrollment of over 220,000 patients in multiple clinical studies. Seventeen studies have been completed to date across all therapeutic classes with no signal for heightened atherosclerotic $\mathrm{CV}$ risk. ${ }^{7}$ However, these studies have provided evidence for a reduction in major adverse cardiovascular events (MACE) along with other benefits. ${ }^{8-11}$ The studies have also identified non-MACE safety signals including increased risks of lowerlimb amputation and heart failure. ${ }^{12-14}$

On October 24, 2018, the FDA held a 2-day Advisory Committee meeting to review the utility and impact of the FDA-required CVOT studies. ${ }^{15,16}$ Several experts from both industry and nonprofit organizations provided commentary

\footnotetext{
' Global Regulatory Science and Policy-NA, Sanofi, Bethesda, MD, USA

Submitted 22-Apr-2019; accepted 06-Jun-2019; published online 6-Jan-2020

Corresponding Author:

Andrew S. Robertson, PhD, JD, Global Regulatory Science and Policy-NA, Sanofi, 4520 East West Highway, Suite 210, Bethesda, MD 208I4, USA.

Email: Andrew.robertson@sanofi.com
} 


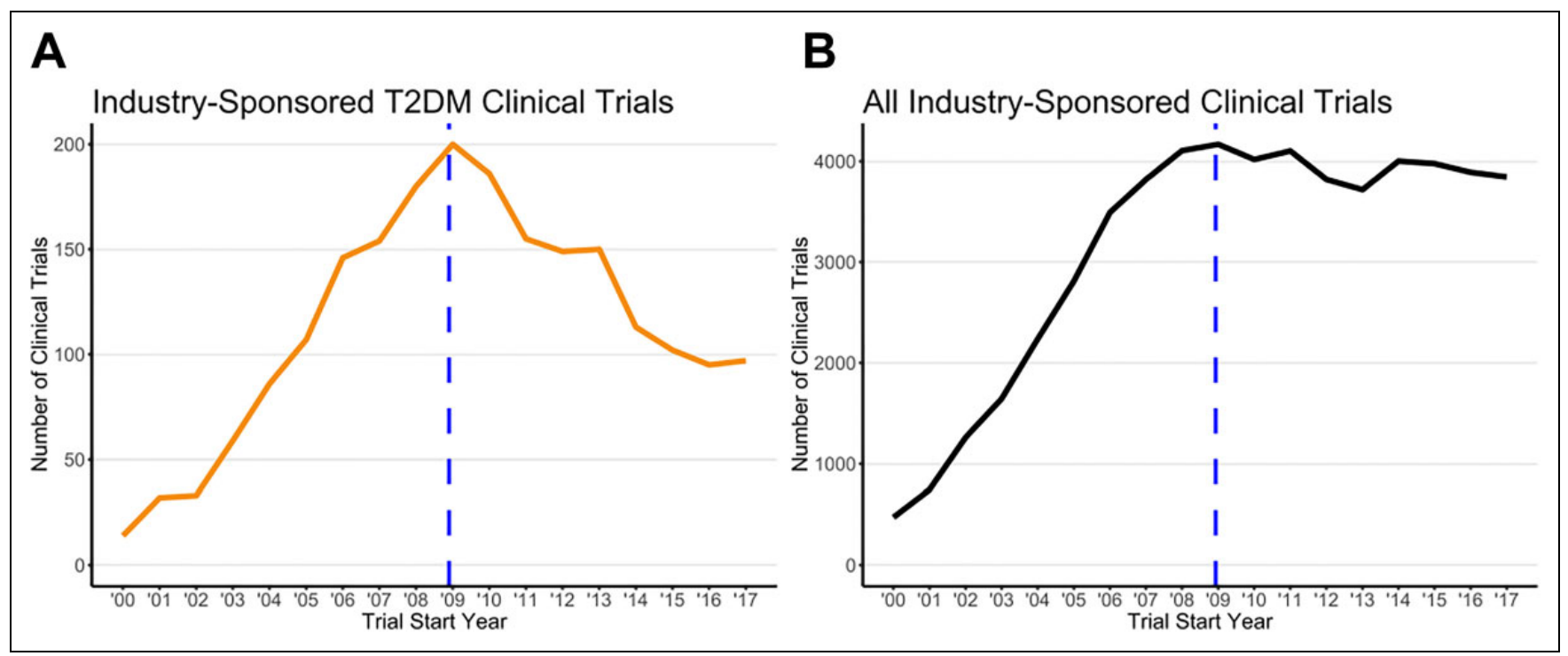

Figure I. Clinical trials by trial start year comparing (A) industrysponsored T2DM trials to $(B)$ all therapeutic area industrysponsored trials. The vertical dashed line marks December 2008.

that these studies posed a significant burden to drug developers, discouraging industry investment in T2DM. ${ }^{17}$ However, this position was primarily based on anecdotal evidence and was dismissed by proponents of the current study requirement. ${ }^{18}$

To better understand the impact that the FDA CVOT study requirement has on industry-sponsored T2DM research, we identified trends in T2DM drug development over the past decade, followed by a deductive analysis of how associated events - specifically, the implementation of the CVOT guidance, the 2008 Global Financial Crisis, the 2010 passage of the Affordable Care Act (ACA), and the rate of novel target discovery in T2DM-may have precipitated any observed changes.

\section{Materials and Methods}

Industry-sponsored phase 1, 2, and 3 T2DM interventional trials initiated between January 1, 2000, and December 31, 2017, were collected using the search term "type 2 diabetes" and relevant variants from www.clinicaltrials.gov $(\mathrm{n}=2058)$. The average T2DM trial enrollment size, where available, was $396.1 \pm 24.8$ patients per study. The year-on-year percentage change in number of initiated clinical studies was calculated to analyze trends in trial initiation rates over time. First, T2DM trends were compared preimplementation of the 2008 FDA-required studies (January 1,2000 , to December 31,2008 ) and post-implementation of the FDA-required studies $(1 / 1 / 2009-12 / 31 / 2017)$, including an aggregate analysis of all T2DM clinical trials initiated during this period, and clinical trials separated by clinical trial phase. Second, initiation rates of clinical studies specific to T2DM were also compared to the rates of clinical trial initiation across all therapeutic areas over the same period January 1, 2000, and December 31, 2017. Third, clinical trial initiation rates were calculated for known T2DM comorbidities ${ }^{19}$ to compare clinical trial investment in a related, diverse selection of therapeutic areas: hypertension $(n=1257)$, obesity $(n=456)$, nephropathy $(n=1760)$, breast cancer $(n=1923)$, and depression $(n=663)$. Comorbidities included within the analysis were selected if they had an incidence in the general population comparable - to or greater than-T2DM, had between 4000 and 10,000 interventional trials (including industry and nonindustry) initiated during the time period sufficient to establish a meaningful trend, used consistent terminology within the www.clinicaltrials.gov database, and had FDA approval of at least 1 product in the respective therapeutic area between 2000 and 2008 and between 2009 and 2017. Fourth, patent information regarding novel antidiabetic agents for the treatment of T2DM was collected from January 1, 1995 through December 31, 2015, online via Google Patents. ${ }^{20}$ All plotting and analyses were performed using R. ${ }^{21}$ Clinical trial data are presented as average year-on-year percentage change \pm standard error. The analysis code, www.clinical trials.gov search parameters, and Google Patent search terms are available on GitHub. ${ }^{22}$

\section{Results}

The analysis shows a significant, recent decline in industryinitiated T2DM studies. Although several events might have contributed to this decline, the CVOT requirement was likely a negative contributing factor.

First, the decline in T2DM clinical trials begins in 2009 (Figure 1A). The number of T2DM clinical studies initiated each year increased between 2000 and $2008(\mu=0.4243 \pm$ 0.1505 ). This trend reversed significantly from 2009 through $2017(\mu=-0.0825 \pm 0.0314)$. Although not causative, this trend reversal correlates closely with the FDA implementation of the 2008 CVOT requirement for T2DM drugs. 


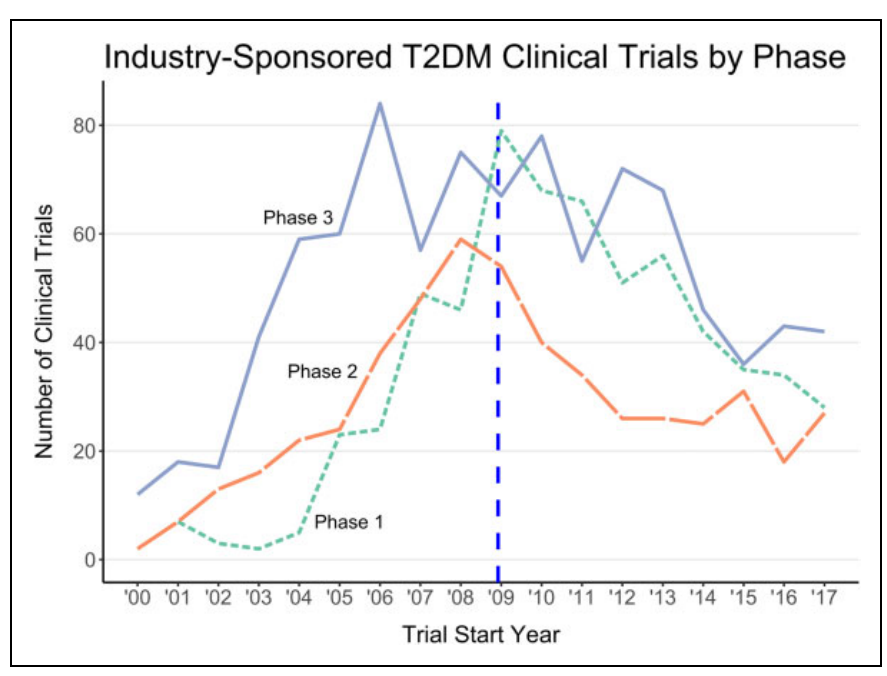

Figure 2. Subanalysis of the number of T2DM clinical trials initiated each year from 2000 to 2017 separated by trial phase. The vertical dashed line marks December 2008.

Second, the number of clinical studies being initiated across all therapeutic areas increased at a rate comparable to the T2DM studies from 2000 to $2008(\mu=0.3246 \pm 0.0762)$ and remained relatively constant from 2008 through 2017 $(\mu=-0.0093 \pm 0.0152$; Figure 1B). This analysis provides information on the possible broad impacts of either the 2008 financial crisis or the 2010 passage of the ACA as a cause for the decline in T2DM research. As observed, the sharp post2008 decline in T2DM clinical trial investment was not reflected in the level of investment in the total number of clinical studies initiated during this period.

Third, the downward trend in T2DM clinical trial initiation was consistent across all trial phases (Figure 2). The largest average year-on-year percentage change was in phase 1 trials $(\mu=-0.0453 \pm 0.1047 ; \mathrm{n}=617)$. The number of initiated phase 3 trials trended downward $(\mu=-0.0308 \pm 0.0840 ; n=506)$ but with high interyear variation. Phase 2 trials trended consistently downward $(\mu=-0.0308 \pm 0.0840 ; \mathrm{n}=928)$.

Fourth, a sample of clinical trials conducted in non-T2DM therapeutic areas did not display the same steep decline seen in T2DM clinical studies (Figure 3). Representative T2DM comorbidities were selected based on the incidence in the general population, a comparable number of interventional trials, use of consistent database terminology, and at least 1 product approval during both study periods (see Methods). The measured average year-over-year changes for the 2009-2017 period increased for breast cancer trials $(\mu=0.0408 \pm 0.0424)$, nephropathy trials $(\mu=0.0355 \pm 0.0314)$, and obesity trials $(\mu=0.0322 \pm 0.0775)$. The number of clinical trials in 2 other therapeutic areas decreased during the 2009-2017 study period, but both had large standard errors (ie, variable trends) and peak trial numbers that were temporally distant from 2009. Depression trials had a negative average year-on-year change $(\mu=-0.0860 \pm 0.1063)$, but the number of depression trials

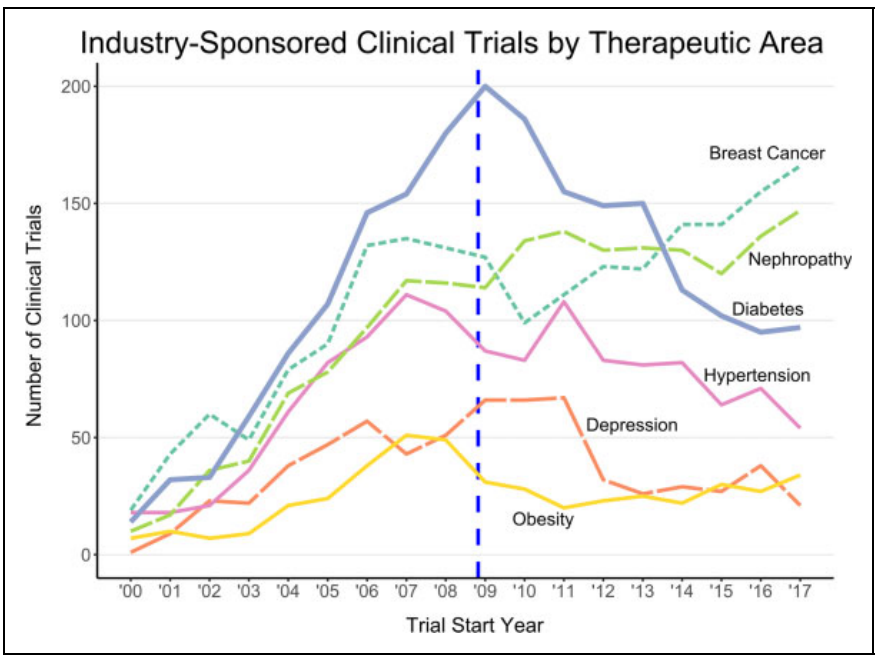

Figure 3. Time series of industry-sponsored clinical study initiation from 2000 to 2017 for several different therapeutic areas with the T2DM ("Diabetes") trials trend bolded. The vertical dashed line marks December 2008.

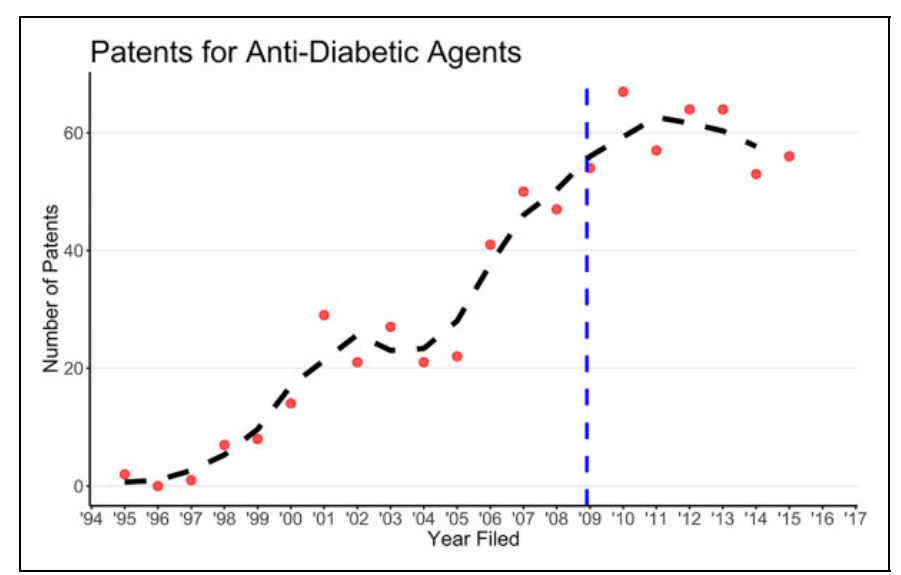

Figure 4. The number of US patents filed for antidiabetic agents each year. The dashed line is the 3-point central moving average. The 20162017 data were omitted from the analysis because of the lag between patent filing and publication. The vertical dashed line marks December 2008.

did not peak until 2011. Similarly, hypertension studies numbers declined post-2008 $(\mu=-0.0422 \pm 0.0669)$, but started declining before 2009, in 2007. Only T2DM initiated trials saw a sharp and steady decrease in number after 2008.

Finally, patent applications for novel antidiabetic agents rose consistently from 1995 through 2011, and remained constant from 2011 through 2015 (Figure 4). The earlier time period starting at 1995 was selected to account for any lag between patent filing and the initiation of clinical trials. This analysis provides a preliminary assessment of whether the decline in T2DM initiated research, particularly early phase 1 trials, might have been affected by a decrease in novel drug or molecular target discovery. This observation, however, suggests that early-stage research 
continued throughout this time period, and cannot account for the decrease seen in clinical-stage drug development.

\section{Discussion}

Together, these observations support the assertion made by patient groups and industry that the 2008 CVOT requirement discourages private sector clinical-stage investment in T2DM research. We acknowledge that the observed decline may be due to a combination of several scientific and economic considerations, and it is reasonable that causative factors may vary with the respective drug, drug target, and business strategy of the sponsoring pharmaceutical company. However, the timing and the nature of the decline in clinical studies supports the deduction that the FDA-required CVOT studies have had a negative industry-wide impact on investment in T2DM drugs.

Despite their large financial and resource costs that may be driving a decrease in private sector investment, CVOTs are not a perfect solution to evaluating the potential for $\mathrm{CV}$ risk in T2DM patients. ${ }^{6,7}$ CVOT sponsors are encouraged to enroll patients with higher $\mathrm{CV}$ risk in order to record a statistically sufficient number of MACEs in a reasonable time frame. ${ }^{23}$ The results from these enriched studies may overstate the risks and/or benefits of the studied therapy and the safety conclusions may not be translatable to the entire intended-to-treat population. Furthermore, CVOTs are typically only short-term studies (generally less than 4 years), leaving the long-term applicability of a treatment's CV risks unknown. The brevity of the studies necessitates continued post-approval pharmacovigilance through standard FDA mechanisms to ensure prolonged CV safety.

Under Section 505(o)(3), FDA has the authority to require sponsors to conduct postmarket studies when the following conditions are met: (1) these studies will help to assess a "serious safety risk," and (2) current adverse event reporting and Sentinel pharmacovigilance systems are not sufficient to conduct the assessment. The past 10 years establish that not all new T2DM drugs carry a "serious safety risk" that would justify a universal requirement that sponsors conduct postmarket studies to exclude CV risk for every new T2DM drug. To date, all completed trials demonstrated that the new drugs studied did not increase the risk of MACE compared to placebo. ${ }^{7}$

Although CVOTs have not identified new CV safety signals, they have provided additional knowledge regarding some aspects of the safety of T2DM treatments. Several of the studied treatments are now known to possess cardioprotective benefits. Additionally, several non-MACE safety signals have been identified, including risks of amputation, hypoglycemia, kidney damage, and heart failure ${ }^{6,7}$ However, these findings fall beyond the original intent behind the 2008 FDA CVOT requirement, and FDA could have adopted a more nuanced and targeted policy approach to ensure appropriate studies were conducted.

As the FDA reconsiders the nature of postmarket requirements for T2DM products, the observations outlined in this article underscore the importance of a greater efficiency in postmarket assessment of CV safety. The FDA currently has the statutory tools, expertise, and technology to follow a risk-based, targeted approach to studying the CV risk of drugs to treat T2DM, consistent with the approach that FDA already uses to evaluate a signal of potential CV risk for all new drugs other than antidiabetic drugs to treat T2DM. Careful assessment of a drug's safety profile based on preapproval CV risk data, use of the Section 505(o)(3) authority to require postmarket studies where deemed necessary, and a mature pharmacovigilance and adverse-event reporting system-including the development of real-world evidence (RWE) pharmacovigilance modeling through the use of Sentinel ${ }^{24,25}$ provide multiple options to help the FDA detect and assess drug risks. CV safety signals could also be more reliably detected by supporting larger, more diverse preapproval studies, as suggested by members of the 2018 FDA Advisory Committee. Where preapproval safety signals of enhanced CV risk exist, implementation of postapproval CVOTs may be warranted, but this should be determined on a product-by-product basis.

\section{Conclusions}

This trend analysis establishes a correlation between the FDA's CVOT requirement and a decrease in industryinitiated T2DM clinical trials. Although not causative, these results support the possibility that the FDA postmarket CVOT requirement is discouraging private sector investment in T2DM drug development.

Our result, coupled with the lack of safety findings from the required CVOT studies, calls into question whether FDA's blanket utilization of their 505(o)(3) authority is the best approach to monitor for elevated safety risks in T2DM. The FDA has extensive experience identifying serious risk signals and monitoring drugs in the post-approval setting. Although constant pharmacovigilance by the FDA ensures patient safety, the FDA should increase options for T2DM patients by encouraging industry-sponsored participation in clinical development of novel T2DM treatments.

\section{Authors' Note}

This article does not contain any studies with human or animal subjects performed by any of the authors.

\section{Acknowledgement}

The authors would like to thank the many experts at Sanofi who provided comments on the manuscript, particularly Alexis Reisin Miller, JD, and Benjamin A. Chacko, PharmD, JD.

\section{Declaration of Conflicting Interests}

Dr Kieffer is a PhRMA Foundation Regulatory Science Fellow working at Sanofi. Dr Robertson is the Head of Regulatory Science and Policy, North America at Sanofi. Sanofi is a pharmaceutical company that develops and markets products to treat patients with type 2 diabetes mellitus. The views expressed in the article are the views of the authors and do not necessarily reflect the views of Sanofi. 


\section{Funding}

No financial support of the research, authorship, and/or publication of this article was declared.

\section{ORCID iD}

Cameron M. Kieffer, PhD (D) https://orcid.org/0000-0001-7509-7503 Andrew S. Robertson, PhD, JD (D) https://orcid.org/0000-0003-2568-5379

\section{References}

1. United States Food and Drug Administration (FDA). Guidance for industry diabetes mellitus - evaluating cardiovascular risk in new antidiabetic therapies to treat type 2 diabetes. https:// www.fda.gov/downloads/Drugs/Guidances/ucm071627.pdf. Published December 2008. Accessed November 12, 2018.

2. Nissen SE, Wolski K. Effect of rosiglitazone on the risk of myocardial infarction and death from cardiovascular causes. $N$ Engl J Med. 2007;356:2457-2471.

3. Home PD, Pocock SJ, Beck-Nielsen H, et al. Rosiglitazone evaluated for cardiovascular outcomes in oral agent combination therapy for type 2 diabetes (RECORD): a multicentre, randomised, open-label trial. Lancet. 2009;373(9681):2125-2135.

4. Mahaffey KW, Hafley G, Dickerson S, et al. Results of a reevaluation of cardiovascular outcomes in the RECORD trial. Am Heart J. 2013;166(2):240-249.

5. US Food and Drug Administration FDA drug safety communication: FDA eliminates the Risk Evaluation and Mitigation Strategy (REMS) for rosiglitazone-containing diabetes medicines. http:// www.fda.gov/Drugs/DrugSafety/ucm476466.htm. Accessed April 19, 2019.

6. Cefalu WT, Kaul S, Gerstein HC, et al. Cardiovascular outcomes trials in type 2 diabetes: where do we go from here? Reflections from a diabetes care editors' expert forum. Diabetes Care. 2018; 41(1):14-31.

7. Regier EE, Venkat MV, Close KL. More than 7 years of hindsight: revisiting the FDA's 2008 guidance on cardiovascular outcomes trials for type 2 diabetes medications. Clin Diabetes. 2016; 34(4):173-180.

8. Marso SP, Daniels GH, Brown-Frandsen K, et al; LEADER Steering Committee; LEADER Trial Investigators. Liraglutide and cardiovascular outcomes in type 2 diabetes. $N$ Engl J Med. 2016;375:311-322.

9. Marso SP, Bain SC, Consoli A, et al. SUSTAIN-6 Investigators. Semaglutide and cardiovascular outcomes in patients with type 2 diabetes. N Engl J Med. 2016;375:1834-1844.

10. Holman RR, Bethel MA, Mentz RJ, et al. EXSCEL Study Group. Effects of once-weekly exenatide on cardiovascular outcomes in type 2 diabetes. N Engl J Med. 2017;377:1228-1239.

11. Zinman B, Wanner C, Lachin JM, et al. EMPA-REG OUTCOME Investigators. Empagliflozin, cardiovascular outcomes, and mortality in type 2 diabetes. $N$ Engl J Med. 2015;373: 2117-2128.

12. Neal B, Perkovic V, Mahaffey KW, et al. CANVAS Program Collaborative Group. Canagliflozin and cardiovascular and renal events in type 2 diabetes. $N$ Engl J Med. 2017;377: 644-657.

13. Scirica BM, Bhatt DL, Braunwald E, et al. Saxagliptin and cardiovascular outcomes in patients with type 2 diabetes mellitus. N Engl J Med. 2013;369(14):1317-1126.

14. White WB, Cannon CP, Heller SR, et al. EXAMINE Investigators. Alogliptin after acute coronary syndrome in patients with type 2 diabetes. $N$ Engl J Med. 2013;369:1327-1335.

15. Schnell O, Rydén L, Standl E, Ceriello A. Updates on cardiovascular outcome trials in diabetes. Cardiovas Diabetol. 2017;16(1):128.

16. Endocrinologic and Metabolic Drugs Advisory Committee; Notice of meeting; establishment of a public docket; request for comments. Fed Regist. 2018;83:47178-47180. https://www.gpo.gov/fdsys/pkg/FR-2018-09-18/pdf/201820233.pdf.

17. Federal Register Docket No. FDA-2018-N-3159-Endocrinologic and Metabolic Drugs Advisory Committee. https:// www.regulations.gov/docket?D=FDA-2018-N-3159. Accessed July, 2019.

18. From goalpost to high hurdle? FDA committee wants "simpler" CV safety standard-but split vote makes FDA's next steps tough. Prevision policy. https:/www.previsionpolicy.com/from-goal post-to-high-hurdle-fda-committee-wants-simpler-cv-safety-stan dard-but-split-vote-makes-fda-s-next-steps-tough. Published October 25, 2018. Accessed November 2, 2018.

19. Handelsman Y, Bloomgarden ZT, Grunberger G, et al. American Association of Clinical Endocrinologists and American College of Endocrinology - Clinical Practice Guidelines for Developing a Diabetes Mellitus Comprehensive Care Plan-2015. Endocr Pract. 2015;21(suppl 1):1-87.

20. Google Patents. patents.google.com. Accessed February 5, 2019.

21. R [computer program]. Version 3.5.1. Vienna, Austria: R Foundation for Statistical Computing; 2018.

22. Kieffer CM. Data-driven-policy. https://www.github.com/Cam Kieff/Data-Driven-Policy. Published April 2019.

23. Caveney E, Turner JR. A review of FDA guidance: understanding the FDA guidance on assessing cardiovascular risks for new antidiabetic therapies. Quintiles. https://pdfs.semanticscholar.org/ f958/a137110410ee240830d33b94fa00dd17df18.pdf. Published 2010. Accessed October 12, 2018.

24. Toh S, Hampp C, Reichman ME, et al. Risk for hospitalized heart failure among new users of saxagliptin, sitagliptin, and other antihyperglycemic drugs: a retrospective cohort study. Ann Intern Med. 2016;164(11):705-714.

25. Aetion announces partnership with the U.S. Food \& Drug Administration and Brigham and Women's Hospital/Harvard Medical School to integrate real-world evidence into regulatory decision-making. Cision PR Newswire. https://www.prnewswire. com/news-releases/aetion-announces-partnership-with-the-usfood-drug-administration-and-brigham-and-womens-hospitalhar vard-medical-school-to-integrate-real-world-evidence-into-regu latory-decision-making-300644080.html. Published May 2018. Accessed April 19, 2019. 